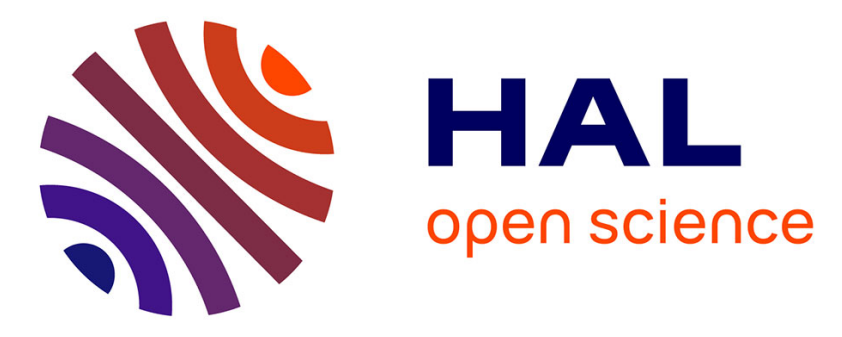

\title{
Collection and exploratory analysis of attitude sensor data in an epilepsy monitoring unit
}

Guillaume Jean-Paul Claude Becq, Stéphane Bonnet, Lorella Minotti, Michel Antonakios, Régis Guillemaud, Philippe Kahane

\section{To cite this version:}

Guillaume Jean-Paul Claude Becq, Stéphane Bonnet, Lorella Minotti, Michel Antonakios, Régis Guillemaud, et al.. Collection and exploratory analysis of attitude sensor data in an epilepsy monitoring unit. Annual International Conference of the IEEE Engineering in Medicine and Biology Society, Aug 2007, Lyon, France. pp.2775-8, 10.1109/IEMBS.2007.4352904 . hal-00124735v2

\section{HAL Id: hal-00124735 \\ https://hal.science/hal-00124735v2}

Submitted on 11 Dec 2007

HAL is a multi-disciplinary open access archive for the deposit and dissemination of scientific research documents, whether they are published or not. The documents may come from teaching and research institutions in France or abroad, or from public or private research centers.
L'archive ouverte pluridisciplinaire HAL, est destinée au dépôt et à la diffusion de documents scientifiques de niveau recherche, publiés ou non, émanant des établissements d'enseignement et de recherche français ou étrangers, des laboratoires publics ou privés. 


\title{
Collection and Exploratory Analysis of Attitude Sensor Data in an Epilepsy Monitoring Unit
}

\author{
Guillaume Becq*, Stéphane Bonnet, Lorella Minotti \\ Michel Antonakios, Régis Guillemaud and Philippe Kahane
}

\begin{abstract}
The aim of this paper is to present the collection of attitude sensor data from an epilepsy monitoring unit and the results of standard exploration using principal component analysis. The collection of data from attitude sensors positioned on three limbs of epileptic patients at their bedside is described. The analysis of the data focuses, on one hand, on motor features extraction from attitude sensor data and on the other hand, on visual segmentation of seizures into events corresponding to motor manifestations classes by an expert. Principal component analysis is then realized over these features and groups of data are localized according to the expert classification. This exploration indicates a possible discrimination between these motor manifestation classes.
\end{abstract}

Index Terms - epilepsy, movement, seizures, attitude sensors, feature extraction, principal component analysis, monitoring, accelerometer, magnetometer.

\section{INTRODUCTION}

$\mathbf{E}$ PILEPSY is one of the most important neurological disorders affecting both adults and children [1]. Epilepsy is characterized by the spontaneous repetition of epileptic seizures which denote brain dysfunction. During an epileptic seizure, an excessive electrical discharge, arising from one brain area, may propagate into other brain areas and induce different clinical manifestations. The chronological organization of these ictal clinical manifestations is actually reflecting the electrical pathway during the discharge. For instance, frontal epileptic seizures are often characterized by the occurrence of one-sided or two-sided motor manifestations of limbs, head or body axis.

The semiological seizure analysis is usually performed in an Epilepsy Monitoring Unit (EMU) by recordings and visualizing seizures with video-EEG recordings. This is essential for the identification of epileptogenic areas in the brain and the comprehension of different clinical and EEG patterns.

Although EEG recording is the gold standard to analyze epileptic seizure, video gives also many clues for the diagnostic. However, video essentially presents qualitative information, which is often hard and long to process. Moreover data visual inspection by EEG technicians is rather subjective and does not allow comparing between different motor patterns. Several solutions have been proposed recently to

Asterisk indicates corresponding author.

This work was supported by the ITS 2006.

G. becq, L. Minotti and P. Kahane are with Inserm U704, Dynamic of neural networks, in the CHU Grenoble, BP217, 38043 Grenoble cedex 9, France guillaume. becq@laposte.net)

S. Bonnet, M. Antonakios and R. Guillemaud are with the CEA/ Leti/DTBS/STD/Lisa, 17 rue des martyrs, 38054 Grenoble cedex, France (stephane.bonnet@cea.fr) quantify movement in an automated, unsupervised and realtime manner.

Different authors have proposed to detect and diagnose epileptic seizures using video analysis with or without markers fixed on human body [2]-[4]. This approach is attractive because it can be based on the routine video-EEG procedure performed in every EMU but a major limitation is that the person (or the markers) should remain in the field-of-view of the camera.

A much powerful approach is based on body-mounted sensors like accelerometers or rate gyroscopes [5]-[8]. MEMS technology is well-suited to monitor epilepsy since it allows quantifying intensity, frequency and duration of movements. Furthermore inertial sensors are well adapted for applications of ambulatory measurement of human movements: high integration, small weight and low cost. As a consequence they can be used outside the clinical setting.

In order to obtain a full characterization of 3D epileptic movements, we propose to combine magnetic sensors with accelerometers into the same attitude sensor module [9]. Magnetometers are primarily intended to characterize movement in the horizontal plane as it can be observed during tonic episodes. Furthermore, these sensors are also sensitive to tremors while being insensitive to accelerations.

This paper aims at showing that quantitative analysis of wrist and head movements, by means of attitude sensors, provides objective measures for the characterization of epileptic seizures and reveals that an automated classification of motor manifestations could be possible. This is done by using principal component analysis (PCA).

In the first section, we describe the clinical environment of this study and then we present the different features that were extracted to characterize epileptic motor manifestations occurring during seizures. Preliminary results are proposed in the last section.

\section{MATERIALS}

The EMU in CHU Grenoble is composed of two bedrooms, each one equipped with Brain Quick video-EEG acquisition system from Micromed [10]. It is composed of an amplifier accepting up to 128 leads with selectable sampling rate from 256 to $1024 \mathrm{~Hz}$ (16 bits resolution). An optical link insures data transmission to a computer in the monitoring room. Audios-videos and EEG recordings are synchronized with Micromed System Plus patient data management system software. In addition to the daily routine, a protocol was 


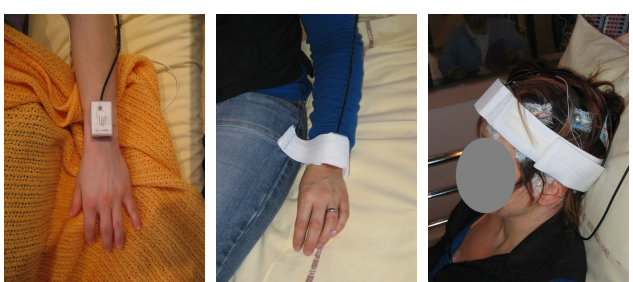

Fig. 1. Attitude sensors (AS) set on patients in an epilepsy unit. On the left, a silicon rectangular parallelepiped was first set on a wrist with adhesive tape. For patient and staff convenience, special dedicated self gripping belts were designed and set on the left wrist (middle), right wrist (not shown) and on the head (right).

elaborated with the medical staff to collect motion information during seizure from body-mounted attitude sensors. To capture upper limb activities, three sensor modules, each containing a tri-axial accelerometer and a tri-axial magnetometer, were used. These attitude sensors (dimension: $50 \mathrm{~mm} \times 30 \mathrm{~mm} \times 15 \mathrm{~mm}$ ) were mounted on both wrist and head segments using straps (Fig. 1). The fabric is washable and well supported by patients.

Every module was linked by a $4 \mathrm{~m}$ wire to a data acquisition system, a so-called Trident system, positioned at the patients bedside. All signals were multiplexed and sampled at $46 \mathrm{~Hz}$ (16 bits resolution) using the Trident system. The energy was supplied by an AC-DC voltage transformer with medical safety approval. Data (18 digital signals) were redirected to a computer in the monitoring room via RS-232 link for recording. An opto-coupling device was introduced to isolate the patient from the computer electrical source coming from the hospital network.

In order to synchronize the video-EEG system and the Trident system, a synchronization was designed. This unit collected a TTL signal $(0-5 \mathrm{~V}, 1 \mathrm{~Hz})$ from the Micromed system as it is currently done currently to drive intermittent photic stimulation (IPS). The synchronization unit provided then a dry contact to the Trident system and this binary information was stored altogether with data.

In practice, the technical staff should activate the IPS at the beginning and end of the recording session. The diagram of the data acquisition process is illustrated in Fig. 2. Finally, video-EEG data and motion data were merged in an offline process into a single file by dealing with time synchronization and resampling.

Data were recorded on 17 patients, 12 women, 5 men, aged from 8 to 45 years during 211 half days of $3 h$ recordings session. Within this period, only 9 patients generated motor seizures with a total of 29 seizures. This corresponds to a ratio of approximately 1.4 episodes per week of working days.

\section{Methods}

\section{A. Motor manifestation classification}

Seizures were visually detected by the medical staff using video EEG and temporally defined by an electrical beginning $t_{0}$, a clinical beginning $t_{1}$ and an electrical end $t_{2}$ as in Fig. 3 .

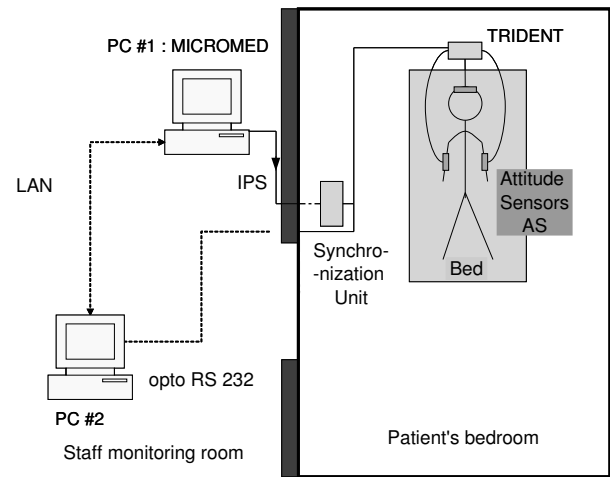

Fig. 2. AS data coming from the different locations are recorded on an isolated PC thanks to the Trident System conversion. In order to synchronize EEG-video data from the Micromed system and AS data, an intermittent photic stimulation (IPS) signal is routed to be used as a marker present in both recordings. Data synchronization is made offline with own dedicated softwares.

Each seizure was visually analyzed by an expert in order to segment each movement from each limb into an event associated to a class of motor manifestation. For each event, we used the classification proposed in [11] as a reference and simplified it into: No movements (NOMVT) no movements observed; Automatisms (AUTO), repetitive automatic movements of limbs and trunks; Clonic manifestations (CLONIC), successive contractions of agonist and antagonist muscles (including myoclonies and clonies); Tonic manifestations (TONIC), sustained contraction of muscle resulting or not in a change of position; Tonico-clonic manifestations (TC), movements combining both clonic and tonic manifestations; Hypermotor manifestations (HYPER), complex movements more or less rapid, involving the proximal segment of limbs or trunk; Versive manifestations (VERSIVE), deviation of the head with or without eyes deviation; Others manifestations (OTHERS), unclassified manifestations (e.g. grinning, paining).

For this study VERSIVE was considered as TONIC, TC was consider as CLONIC and data of the OTHERS class were discarded. A number of artifacts generated by the medical staff for clinical evaluation or patient's care were removed for data cleaning.

\section{B. Feature extraction}

1) General concept: AS data coming from the different events were collected into a database. They follow a feature extraction process in order to obtain for each event a vector containing a set of angular, dynamic, spectral and wavelet features supposed to be relevant for each class an possibly useful for classification. A block diagram of the feature extraction process is presented Fig. 4.

The principal asset of our method based on AS data is the evaluation of the principal Euler angle and features extraction from it.

2) Principal Euler angle computation: Using both vector observations $\overrightarrow{A c c} / b$ and $\overrightarrow{M a} g_{/ b}$ and combining them into $\overrightarrow{A S} / b$, it is possible to estimate the sensors attitude with 


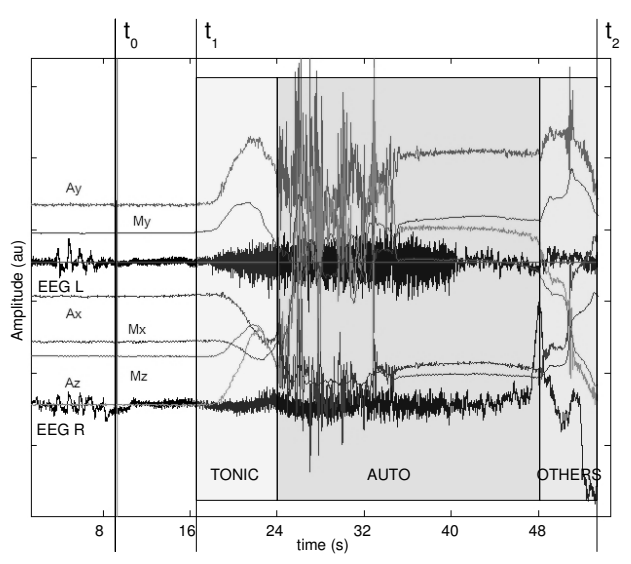

Fig. 3. Data from AS and EEG are merged together. EEG enables an expert to localize the seizure defining: $t_{0}$ the electrical beginning of the seizure, $t_{1}$ the clinical beginning of the seizure, and $t_{2}$ the end of the seizure. Visual inspection with video enables an expert to segment the seizure into different events. Data presented here come from the left wrist. Three events corresponding to tonic, automatism and others manifestation are succeeding one after another.

respect to a frame of reference [9] ${ }^{1}$. In this study, we chose to use the Euler angle/axis representation: a rotation from the reference frame to the current frame is performed about the unit axis $\vec{u}$ through angle $\theta$. A sample to sample or instantaneous rotation was determined using: $\theta\left(t_{i}\right)=$ $f_{\Theta}\left(A \vec{S}_{/ b}\left(t_{i}\right), A \vec{S}_{/ b}\left(t_{i-1}\right)\right)$ with $t_{i}=t_{s}+i / F_{s}, t_{e}=t_{s}+$ $(N-1) / F_{s}, t_{s}$ start time of the event, $t_{e}$ end time of the event, and $f_{\Theta}$ an estimation function based on least min squared minimization [9].

3) Angular features: Angular features like in [8] using gyroscopes for measuring rotation rates were computed: the root mean square of the signal, referred as the mobility by Salarian, Mobility $=\sqrt{\frac{1}{(N-1)} \sum_{i=1}^{N-1} \theta^{2}\left(t_{i}\right)}$; from the cumulated rotation $\Theta\left(t_{j}\right)=\sum_{i=1}^{j} \theta_{s}\left(t_{i}\right)$ by removing instantaneous rotations $\theta_{s}$ with angular velocities $\theta(t) * F_{s}$ fewer than $10^{\circ} / \mathrm{s}$, the median value med $_{\Theta}$ and the range of values Range.

4) Dynamical features: We used the norm of acceleration $\|\overrightarrow{A c} c\|(t)$ to compute dynamical features as a good candidate to discriminate movements versus no movements $\|\overrightarrow{A c c}\|(t)=\sqrt{A c c_{x}(t)^{2}+A c c_{y}(t)^{2}+A c c_{z}(t)^{2}}$. The entropy of the norm of acceleration, $E n_{\|\overrightarrow{A c}\| \|}=$ $-\sum_{i} h_{i} \log \left(h_{i}\right)$, was also retained with a quantification of 16 bits between 0 and $3 G$ to compute $h_{i}=n_{i} / N$, with $n_{i}$

\footnotetext{
${ }^{1}$ The measurement model for the accelerometer is given by: $\overrightarrow{A c c}=\vec{g}-$ $\vec{\gamma}+$ noise. The components of this vector quantity are resolved in the bodyfixed frame: $\overrightarrow{A c c} / b=\left(A c c_{x}, A c c_{y}, A c c_{z}\right)^{t}$. The measured acceleration signal is a combination of a low-frequency component due to the sensor orientation with respect to gravity $(\vec{g})$ and a high-frequency component due to the sensor movement itself $(\gamma)$. For slow movements, the accelerometer is principally acting as an inclinometer and the norm of the accelerometer is close to $1 \mathrm{~g}$. The separation between the two components is approximately achieved by low-pass filtering (Butterworth, 4th order, cut-off frequency: $0.5 \mathrm{~Hz}$ ). The measurement model for the magnetometer is simplified into: $\overrightarrow{M a g}=\overrightarrow{M a} g_{\text {earth }}+$ noise. Where $\overrightarrow{M a} g_{\text {earth }}$ denotes the Earth magnetic field vector. The components of this vector quantity are resolved in the bodyfixed frame: $\overrightarrow{M a g} g_{/ b}=\left(\operatorname{Mag}_{x}, \operatorname{Mag}_{y}, \operatorname{Mag}_{z}\right)^{t}$.
}

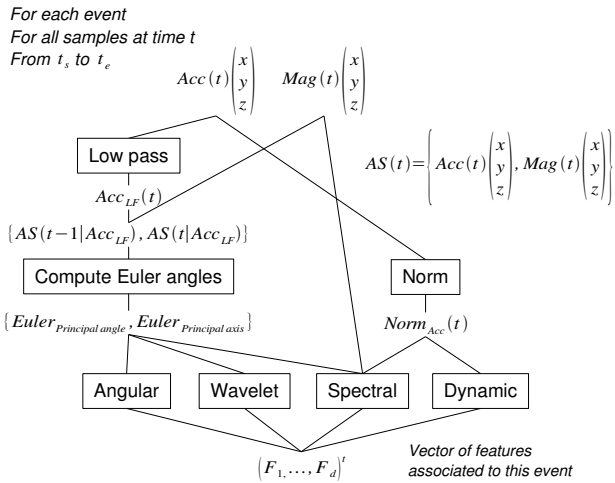

Fig. 4. Feature extraction block diagram. Data from accelerometer $\overrightarrow{A c c}(t)$ and from magnetometer $\overrightarrow{M a g}(t)$ are grouped together to form an attitude sensor vector $\overrightarrow{A S}(t)$. Filtering, Euler angles computation and norm computation leads to new variables for features extraction. This is done for data of each event, resulting in one vector of features associated to each event.

the number of samples at level $q_{i}\left(n_{i}=\#\left(\|\overrightarrow{A c c}\|(t)=q_{i}\right)\right)$, $N$ the number of samples in the event and $h_{i} \log \left(h_{i}\right)=0$ for $h_{i}=0$.

5) Spectral features: Spectral features were evaluated using the spectral edge frequency (SEF), indicating the frequency value for which $\alpha \%$ of the spectral power is obtained for 10 and $95 \%$ (respectively $S E F_{10}$ and $S E F_{95}$ ). Features for $\Theta$ and medio-lateral axis component for $A c c$ and $\mathrm{Mag}\left(\mathrm{Acc}_{m l}, \mathrm{Mag}_{m l}\right)$ were computed.

6) Wavelet features: Wavelet features were evaluated using a ratio of wavelet coefficients in different scales bands. Coefficients came from a continuous wavelet transform, $C_{x}(a, b)=\int_{-\infty}^{\infty} x(t) \psi(a, b, t) d t, a$ being a scale parameter and $b$ a lag parameter, using a Morlet atom wavelet $\psi(t) \sim$ $\exp \left(-x^{2} / 2\right) \cos (5 x)$, with the Matlab wavelet toolbox [12]. The different scales retained where chosen to correspond to the pseudofrequencies grouped into three different bands $\delta_{3}=\{23,20,17,14,12,10,9\} \mathrm{Hz}, \delta_{2}=\{8,7,6,5$, $4,3,2\} H z, \delta_{1}=\{1.25,1,0.75,0.5,0.25,0.1,0.05\} \mathrm{Hz}$. Features for $A c c_{m l}, \mathrm{Mag}_{m l}$ and $\Theta$ were computed.

\section{Principal component analysis}

Features data were standardized using the transformation $Y_{j}=\frac{X_{j}-\mu_{j}}{\sigma_{j}}$ with $X_{j}$ vectors made from the samples of the $j$ th feature, $\mu_{j}$ and $\sigma_{j}$ the mean and standard deviation of these samples. 30 uniform random samples from each sample were introduced into the database for data regularization. A principal component analysis was realized in order to find the best linear combinations of all features retaining the best singular values of the correlation matrix. This often results in better separation of data in the new basis and indicates possible discrimination.

\section{RESULTS}

226 manifestations events were scored, representing in each class: 42 NOMVT, 25 AUTO, 17 CLONIC, 70 TONIC, 21 TC, 20 HYPER, 11 VERSIVE, 20 OTHERS. 


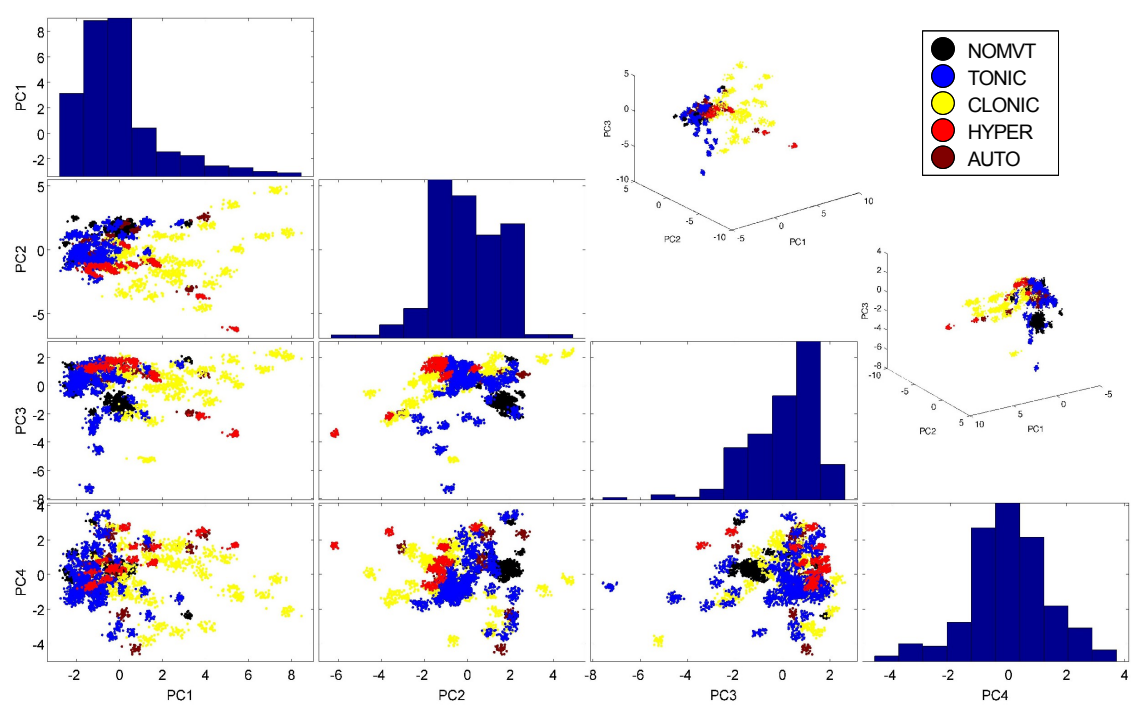

Fig. 5. Data projection on the four first principal components. In the diagonal, figure indicates histograms of data for this component. Each data corresponding to one event is represented by its new coordinates (scores) in the principal components basis. A color indicates the motor manifestation class of the event. 30 random samples from each original sample were generated to simulate densities. 3D scatters in a 3 first components basis are represented on the right top with different fields of view.

Results of the principal component analysis can be represented by the scores of each event in the new basis constituted with the principal component. A cloud representation of the new data point with projection over the four first principal components is given Fig. 5. Each data is represented by a circle with a color indicating the motor manifestation class of the associated event.

By visualizing this representation, it is clear that projections on the first, second and third components indicate a possible discrimination between classes TONIC, CLONIC, HYPER and NO MVT. This is emphasized by 3D scatter plots from different points of view. Class AUTO seems to be difficult to discriminate since lot of its samples are melted with other classes. Projections on the 4th components do not seem to introduce better discrimination for one class or another.

Histograms on the first and second components indicate a large proportion of TONIC classes separated from other classes (especially CLONIC classes). Histogram on the third component indicates a little evidence for class NOMVT vs others (left part of the histogram). Histogram on the fourth component indicates also that classes are not well separated with this projection.

\section{DISCUSSION}

Using AS data and applying PCA indicates that an automatic classification of features extracted from this data into motor manifestations relative to epilepsy can be possible.

The discrimination between classes suffers principally from the proximal nature of some manifestations and on the weak discriminative power of some retained features that must be investigated.

We expect to learn classifiers with the best features. This will allow us to obtain automatic succession of motor manifestations classes and generate graphs as a tool for neurologists to characterize the stereotaxic evolution of motor manifestations during seizures.

\section{ACKNOWLEDGMENT}

The authors would like to thank the medical staff, Patricia Boschetti, Véronique Dorlin, Martine Juillard and the patients that participate to the study.

\section{REFERENCES}

[1] P. Thomas and A. Arzimanoglou, Epilepsies, 3rd ed. Paris, France: Masson, 2003.

[2] N. B. Karayiannis et al., Quantifying motion in video recordings of neonatal seizures by robust motion trackers based on block motion models, IEEE Trans. on Biomed. Eng., 52(6): 1065-1077, 2005

[3] N. B. Karayiannis et al., Automated detection of videotaped neonatal seizures of epileptic origin, Epilepsia, 47(6): 966-980, 2006

[4] Z. Li, A. M. da Silva and J. P. da Silva Cunha, Movement quantification in epileptic seizures: a new approach to video-EEG analysis, IEEE Trans. on Biomed. Eng., 49(6): 565-573, 2002

[5] J. D. Frost, Triaxial accelerometry: a method for quantifying tremor and ataxia, IEEE Trans. on Biomed. Eng., vol. 25, p. 17-27, 1978

[6] B. Kemp, J. M. W. Janssen and B. van der Kamp, Body position can be monitored in $3 D$ using miniature accelerometers and earth-magnetic field sensors, Electroenceph. and Clin. Neurophys., 109:484-488, 1998

[7] T. M. E. Nijsen et al., The potential value of three-dimensional accelerometry for detection of motor seizures in severe epilepsy, Epilepsy and Behavior, 7:74-84, 2005

[8] A. Salarian et al., An ambulatory system to quantify bradykinesia and tremor in parkinson's disease, 4th ann. IEEE Conf. on Information Technology Applications in Biomedicine, p. 24-26, April 24-26, 2003

[9] S. Bonnet and R. Héliot, A magnetometer-based approach for studying human movements, IEEE Trans. on Biomed. Eng., accepted for publication, 2007

[10] Micromed s.r.l., via Giotto,2 - 31021 Mogliano Veneto (TV), Italy, http://www.micromed-it.com/

[11] H. Lüders et al., Semiological Seizure Classification, Epilepsia, 39(9): 1006-1013, 1998

[12] Matlab®, The MathWorks Inc., 3 Apple Hill Drive Natick, MA, http://www. mathworks.com/ 